\title{
Detection of Trabeculae and Papillary Muscles in Cardiac MR Images
}

\author{
LJ Spreeuwers $^{1,2}$, SJ Bangma ${ }^{1}$, RJHW Meerwaldt ${ }^{1}$, EJ Vonken $^{1}$, M Breeuwer $^{3}$ \\ ${ }^{1}$ University Medical Center Utrecht, Netherlands \\ ${ }^{2}$ University of Twente, Enschede, Netherlands \\ ${ }^{3}$ Philips Medical Systems, Best, Netherlands
}

\begin{abstract}
With the improvement of the quality of MR imagery, more and more details become visible. Only 5-10 years ago cardiac images of the heart were still so unsharp that finer details of the heart like the papillary muscles and the trabeculae were hardly visible and it was simply impossible to determine their outlines with any measure of accuracy. With the improved image quality it becomes feasible to extract information about these small structures. Studying the operation of these tiny muscles can be very useful for further analysis of the heart function and diagnosis of heart diseases. Until now very little literature existed on the study of these structures using cardiac MR, so with this publication we are riding the front of the wave. We conducted two pilot studies to investigate the feasibility of detection of papillary muscles in the left ventricular blood volume and to obtain a measure of the trabeculation of the right ventricle. The latter was also investigated as a diagnostic criterion for Arrhythmogenic Right Ventricular Dysplasia (ARVD).
\end{abstract}

\section{Introduction - small structures}

The work presented in this article aims to perform measures on the small muscular structures in the heart, in particular the papillary muscles and the trabeculae, using MR imagery. Until recently, MR images did not provide sufficient detail to perform these measures, but with the increasing quality of the acquisition it becomes possible to segment these small structures. In this section, first a description is given of the anatomy of the papillary muscles and the trabeculae. The next two sections describe the segmentation of the papillary muscles in the left ventricle and measurements on the trabeculae in the right ventricle. Both sections describe the method applied as well as the results obtained. Finally, in section 4 conclusions are presented.

\subsection{Papillary muscles}

The papillary muscles can be found in the left and right ventricles. These muscles project from the ventricular walls and are roughly conical in shape with a rounded end. Their purpose is to keep the atrioventricular valves closed when the ventricles contract. This prevents the blood from flowing back into the atria. The leaflets of the valves are connected to the papillary muscles by the chordae tendinae. Most chordae tendinae arise from the tip of the papillary muscles, but some also arise from near the base. The papillary muscles contract a fraction earlier than the ventricle itself in order to prevent the valves from being forced open by the rising pressure in the ventricle. The papillary muscles and chordae tendinae and the open and closed valves are shown in fig. 1. This figure was taken from [1].

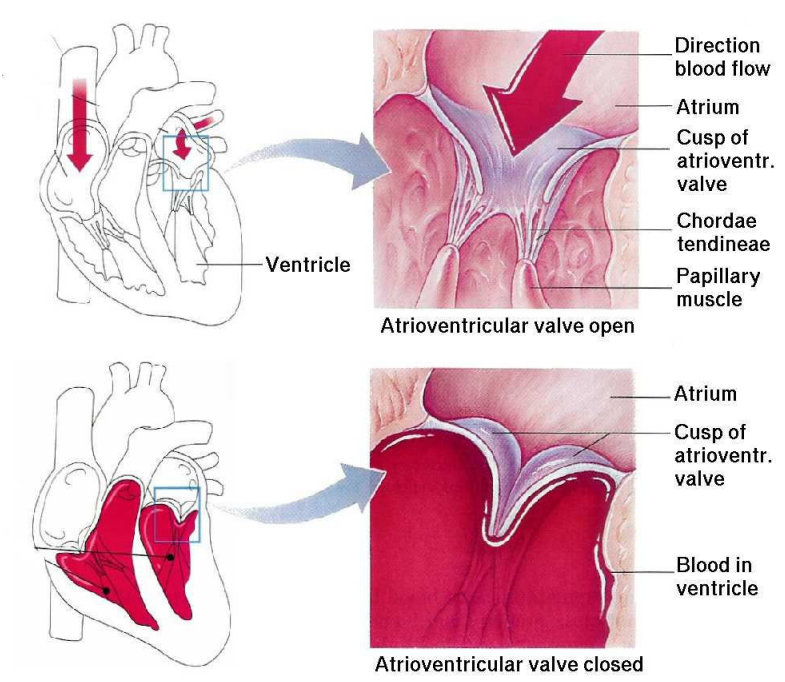

Figure 1. Papillary muscles and chordae tendinae. Top: open valves, blood flows into ventricle; Bottom: valves closed and papillary muscles contract to prevent prolapse of the leaflets in the atrium

Since we concentrated on segmenting papillary muscles in the left ventricle, some more details will be given here 
(for further details, see e.g. [2, 3, 4]). The left ventricle has two papillary muscles: the anterolateral and the posteromedial papillary muscle. The chordae tendinae from both papillary muscles diverge and are connected to both leaflets of the mitral valve. Instead of papillary muscles, it would be better to speak of two groups of papillary muscles. The anterolateral group usually consists of a single muscle, but it can also consist of two or three muscle heads or bellies. The posteromedial group usually consists of two, but can also consist of one, three or even more muscle heads or bellies. The origin of the papillary muscles is usually located near the middle third of the left ventricle wall and they project into upper third of the left ventricle, the main axis running parallel to that of the ventricular cavity. The attachment of a papillary muscle to the wall varies from only at the base to along their entire length. Measurements of the length of the papillary muscles vary from 2 to $3.5 \mathrm{~cm}$ and their thickness is in the order of $0.5-1 \mathrm{~cm}$ [5]. The length and thickness of the chordae tendinae are in the order of $1-2 \mathrm{~cm}$ and $0.2-1 \mathrm{~mm} \mathrm{[6]} \mathrm{which} \mathrm{means} \mathrm{that} \mathrm{in}$ MR images used, they are not visible, because the in-plane resolution is around $2 \mathrm{~mm}$.

As a conclusion we can state that papillary muscles are very divers in shape and position, which complicates segmentation.

\subsection{Trabeculae}

The second tissue type we investigated are the trabeculae, which can be found in both the right and the left ventricle. There are two types of trabeculae: the trabeculae carneae, which consist of bands of muscle lining the inside of the ventricles and the trabecula septomarginalis or moderator band that consist of bands of muscle that traverse through the ventricle. The trabeculae carneae can be found in both ventricles and are thought to reduce the turbulence of blood during systole. The moderator band lies inside the right ventricle and carries the purkinje fibres (part of the conducting system of the heart) to the outer wall of the ventricle. Sometimes the papillary muscles are regarded as a third type of trabeculae. The trabeculae in the right ventricle are shown in figure 2, which was taken from [7]. Like the papillary muscles, the trabeculae are very divers in shape and position.

An increase in the amount of trabecular tissue and an increase in the thickness of these trabeculae constitutes to an increased trabeculation. Increased trabeculation has been witnessed in several studies in people with Arrhythmogenic Right Ventricular Dysplasia (ARVD). ARVD is a heart disease which manifests itself by the replacement of muscle tissue within the wall of the heart with fatty and fibrous tissue. It frequently causes live threatening arrhythmias in patients and is considered to be the primary cause of cardiac failures in people under the age of 35 . The dis-

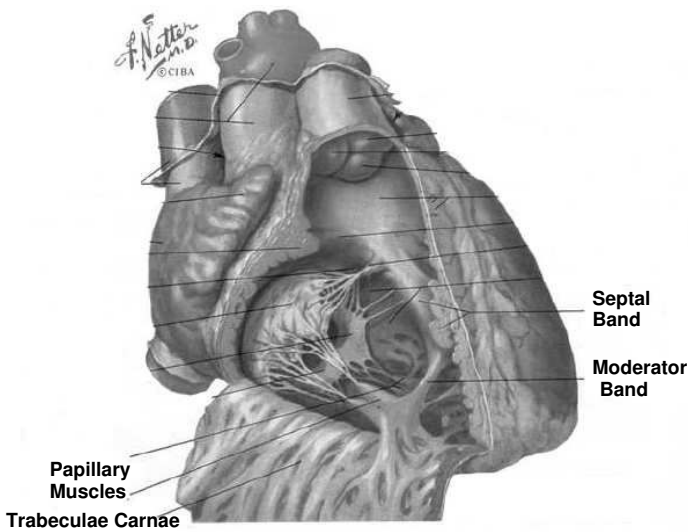

Figure 2. Trabeculae in the right ventricle

ease has many symptoms which are not necessarily present in all patients. Because no set of symptoms is $100 \%$ discriminant to the disease, ARVD is very hard to diagnose. Diagnosis to this date is based on a set diagnostic criteria described by McKenna et al. [8]. Unfortunately these criteria require intensive and invasive testing of subjects which includes biopsies of the cardiac wall. Hence the importance of obtaining an estimate of increased trabeculation using a non-invasive method like MR.

\section{Segmentation of papillary muscles}

\subsection{Literature}

Only a few works have touched on the subject of segmenting the papillary muscles even though they are often mentioned as an important factor complicating the segmentation of the myocardium. To the best of our knowledge, no literature exists on complete segmentation of the papillary muscles in cardiac MR images. Kaushikkar et al [9] describe an adaptive threshold based method for segmentation of the left ventricular blood pool in 2-D shortaxis slices which is able to segment papillary muscles that are completely surrounded by blood. Makowski et al. [10] describe an active contour method for extraction of structures in cardiac MR images which again is only capable of extracting papillary muscles that are completely surrounded by blood.

\subsection{Image data}

The short-axis data sets are cine MRI sequences, recorded with a 1.5 Tesla Philips Gyroscan scanner using the balanced FFE protocol. They consist of 9 to 12 slices of $8 \mathrm{~mm}$ thick and 20 phases. The slices cover an area of $400 \times 400 \mathrm{~mm}$ and are 256x256 pixels large. An example of three consecutive short-axis slices is shown in figure 3 . In these images the myocardial boundaries are shown as 
well. The papillary muscles are the dark spots in the left (and right) ventricular blood volume.
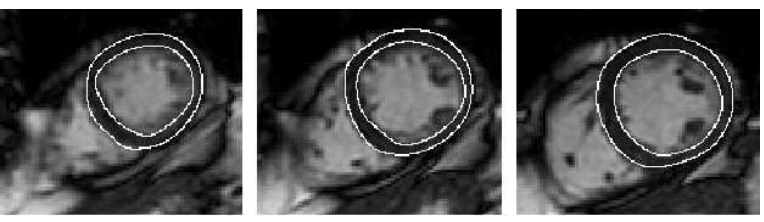

Figure 3. Short-axis MR slices with papillary muscles

\subsection{Segmentation method}

The method we propose to segment papillary muscles in the left ventricular blood volume is a constrained region growing method. The region growing takes place in a 2D slice and the results are propagated to the neighbouring slices where the region growing process is repeated. The initial slice and the seed points for the region growing process are selected manually and we assume that the myocardial boundaries of the left ventricle are given (like in fig.3). The region growing starts at the seed points and then adds pixels with a grey level below a given threshold (papillary muscles are darker than the surrounding blood). However, because near the myocardium, due to partial volume effects, the intensity decreases significantly this would result in serious over segmentation. Therefore, a constrained region growing method was used for which the threshold decreases near the myocardial boundary:

$$
T(d)=T_{\infty} \frac{1}{e^{1+k\left(d_{0.5}-d\right)}}
$$

Where $T$ is the threshold, $d$ is the distance of the pixel to the myocardium, $T_{\infty}$ is the threshold at infinite distance from the myocardium, $k$ determines the steepness of the decrease of the threshold and $d_{0.5}$ is the distance where the threshold has decreased to $50 \%$ of $T_{\infty}$. The propagation of a segmentation in one slice to neighbouring slices is done by dilating the segmentation $N_{d}$ times and making all found pixels potential seed pixels in the neighbouring slices. The dilation is important if the papillary muscle splits into multiple heads. The threshold $T_{\infty}$ is determined from the grey levels of blood: $l_{b}$ and the seed points of the papillary muscle region growing: $l_{p}$, according to:

$$
T_{\infty}=I_{p}+\alpha\left(I_{b}-I_{p}\right)
$$

The parameters of the method were determined using a training set of 10 manually segmented data sets. The resulting parameters are given in table 1 .

\subsection{Segmentation results}

Evaluation of the segmentations was done using 10 independent data sets. Each data set was segmented manually

\begin{tabular}{|r|r|r|r|}
\hline alpha & $k$ & $d_{0.5}$ & $N_{d}$ \\
\hline 0.35 & 5 & 2.75 & 2 \\
\hline
\end{tabular}

Table 1. Parameter values obtained using training

twice by one observer and once by a second observer. The results of the method appeared to be very close to the inter observer variability and smaller than the intra observer variability. Using the segmentations of all slices, we were also able to reconstruct 3-d models of the papillary muscles. In fig. 4 examples of such a reconstruction are given.

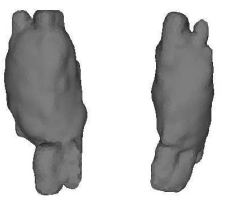

Figure 4. 3-d reconstruction of papillary muscles

\section{Measurements on trabeculation}

\subsection{Literature}

Few experiments have been performed on the usefulness of MRI in imaging right ventricular wall abnormalities. However, the 1998 task force of the European society of cardiology lists MRI as probably the best imaging technique for demonstrating structural and functional abnormalities of the right ventricle. Aviram et al. [11] report on a study of the use of MRI in the diagnosis of ARVD. They specify five criteria among which heavy right ventricular trabeculation, but only evaluate this qualitatively.

\subsection{Image data}

Similar short-axis data as for the segmentation of papillary muscles was used. Again balanced FFE protocols were used and 12 slices of $8 \mathrm{~mm}$ thick were recorded. This time the number of phases was either 20 or 50 and the pixel size varied from 1.37 to $1.76 \mathrm{~mm}$. The image size was $256 \times 256$ pixels. For this study 8 data sets were available of which 4 from ARVD patients. Figure 5 shows an example of the right ventricle and the trabeculae (black blobs).

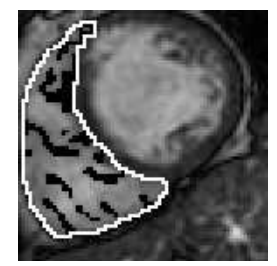

Figure 5. Trabeculae in the right ventricle (black blobs) 


\subsection{Method}

From manual segmentations and automatic segmentations using simple thresholding, thresholding with a partial volume model and fuzzy clustering, a number of features were extracted and used to classify the slices of the data sets and the whole complete sets. Among these features were volume measures, like total trabeculae volume, shape measures, like average length and width of clusters and texture measures using co-occurrence matrices. The texture features are extracted directly from the images so no prior segmentation is required. Two classifiers were used to classify combinations of these features: a Bayes classifier and a nearest neighbour classifier. Training and evaluation of the classification results was done using the leave one out approach.

\subsection{Results}

The results of the automatic segmentations were compared to the manual segmentations using the so-called Dice Similarity Measure (DSC). The fuzzy clustering method gave results that were most comparable to the manual segmentations. The classification results based on volume features extracted from the manual segmentations were quite encouraging with a detection rate of $87.5 \%$. The shapebased features performed much worse with a detection rate of only $25 \%$. The volume and shape based features of the automatic segmentations gave rather disappointing classification results compared to those of the manual segmentations. The classification results based on the texture features are quite reasonable with $69 \%$ correct classification on all slices. The classification results are given in table 2 . These compare favourable with the scores based on traditional ARVD criteria (around 75\%). However, we should take into account the limited number of data sets.

\begin{tabular}{|l|l|r|r|}
\hline segmentation & features & Bayes & $\mathrm{NN}$ \\
\hline manual & volume & 0.88 & 0.88 \\
manual & shape & 0.25 & 0.25 \\
threshold & volume & 0.75 & 0.63 \\
threshold & shape & 0.25 & 0.88 \\
threshold part. vol. & volume & 0.25 & 0.50 \\
threshold part. vol. & shape & 0.13 & 0.25 \\
fuzzy clustering & volume & 0.50 & 0.25 \\
fuzzy clustering & shape & 0.20 & 0.47 \\
& texture & 0.69 & 0.69 \\
\hline
\end{tabular}

Table 2. ARVD classification results

\section{Conclusions}

We presented the first results of our attempts to segment papillary muscles and to measure trabeculation in the right ventricle. Using the segmentations we were able to reconstruct a 3-d model of papillary muscles in the left ventricle. On a small data set the measure for trabeculation in the right ventricle appeared a promising indicator for ARVD.

\section{References}

[1] Marieb E. Human Anatomy \& Physiology. 4th edition. Menlo Park, California: Addison Wesley Longman/Benjamin Cummings, 1998.

[2] Estes Jr. E, Dalton F, Entman M, Dixon II H, Hackel D. The anatomy and blood supply of the papillary muscles of the left ventricle. American Heart Journal 1966;71(3):356362.

[3] Ranganathan N, Burch G. Gross morphology and arterial supply of the papillary muscles of the left ventricle of man. American Heart Journal 1969;77(4):506-516.

[4] Victor S, Nayak V. Variations in the papillary muscles of the normal mitral valve and their surgical relevance. Journal of Cardiac Surgery 1995;10(5):597-607.

[5] Roberts W, Cohen L. Left ventricular papillary muscles. description of the normal and a survey of conditions causing them to be abnormal. Circulation 1972;46(1):138-154.

[6] Boltwood C, Chuwa T, Wong M, Shah P. Quantitative echocardiography of the mitral complex in dilated cardiomyopathy: the mechanism of functional mitral regurgitation. Circulation 1983;68(3):498-508.

[7] Netter F. Atlas of Human Anatomy. Ardsley, USA: CIBAGEIGY Corporation, 1989.

[8] McKenna W, Thiene G, Nava A, Fontaliran F, BlomstromLundqvist C, Fontaine G, , Camerini F. Diagnosis of arrhythmogenic right ventricular dysplasia/cardiomyopathy. British Heart Journal 1994;71:215-218.

[9] Kaushikkar S, Li D, Haacke E, Dávila-Román V. Adaptive blood pool segmentation in three dimensions: application to mr cardiac evaluation. Journal of Magnetic Resonance Imaging 1996;6(4):690-697.

[10] Makowski P, Sorensen T, Therkildsen S, Materka A, Stodkilde-Jorgensen H, Pedersen E. Thow-phase active contour method for semiautomatic segmentation of the heart and blood vessels from mri images for $3 \mathrm{~d}$ visualization. Computerized Medical Imaging and Graphics 2002; 26(1):9-17.

[11] Aviram G, Fishman J, Young M, Redha E, Biliciler-Denktas G, Rodriguez M. MR evaluation of arrhythmogenic right ventricular cardiomyopathy in pediatric patients. American Journal of Roentgenology 2003;180(4):1135-1141.

Address for correspondence:

L.J. Spreeuwers

Signals and Systems Group, Electrical Engineering

University of Twente, P.O.Box 217

7500 AE Enschede, The Netherlands

L.J.Spreeuwers@utwente.nl 